\title{
Modeling and mitigation of fiber nonlinearity in wideband optical signal transmission
}

\author{
Daniel Semrau*, Eric Sillekens, Polina Bayvel, and Robert I. Killey \\ Optical Networks Group, University College London, Department of Electronic and Electrical Engineering, Roberts Building, Torrington Place, London WC1E \\ 7JE, UK \\ *Corresponding author: uceedfs@ucl.ac.uk
}

\begin{abstract}
The adoption of open optical networks (OONs) requires the development of open and effective network planning tools, enabling the use of multi-vendor or white box transport solutions. Such tools for studying and planning optical networks must be able to take into account the physical layer impairments, including fiber nonlinearity. The use of wideband wavelength division multiplexing in OONs, with channel frequencies extending across the short-, conventional- and long-bands (S, C and L-bands) and beyond, offers a pathway to increasing data rates through the installed fiber infrastructure. However, achievable information rates are limited by the resulting signal distortion due to fiber nonlinearity as signal bandwidths are increased, in particular inter-channel stimulated Raman scattering (ISRS). In this paper, we describe the nonlinear effects observed in wideband transmission systems, and review recently-developed analytical tools, based on the Gaussian noise (GN) model of nonlinear interference with the inclusion of ISRS. Using the ISRS GN model, we assess the impact of fiber nonlinearity on the achievable information rates in transmission systems with bandwidths of up to $12 \mathrm{THz}$. We demonstrate the use of the model in the optimization of launch power spectral profiles for a variety of dynamic gain equalizer arrangements in a $1000 \mathrm{~km}$ standard single-mode fiber link, using particle swarm optimization and the steepest descent algorithm. Such nonlinear models and optimization methods could be applied in OON planning tools, for example in optical link emulators to estimate quality-of-transmission (QoT) and data throughput, and in impairment-aware software-defined network control and management.
\end{abstract}

\section{INTRODUCTION}

Exponentially increasing data traffic in optical fiber communication networks around the world is resulting in the adoption of a number of strategies to satisfy demand, including wideband wavelength division multiplexing (WDM), space division multiplexing (SDM) and spectrally-efficient modulation formats.

Wideband WDM technologies allow increased fiber link and network capacities, and are being used by network operators aiming to fully exploit their existing single-mode fiber (SMF) infrastructure to optimize their returns on CAPEX. Wideband WDM is also compatible with space division multiplexing, offering a path to future link capacities in the petabit/s range.

Most currently installed systems use the conventional-band (C-band, over the wavelength range 1530 - $1565 \mathrm{~nm}$ ) with a bandwidth of just $35 \mathrm{~nm}$ or $4.4 \mathrm{THz}$. Some systems are now being deployed using both the conventional- and long-bands (C+L-band, 1530 - $1625 \mathrm{~nm}$ ), with signal bandwidths of up to 11.5 $\mathrm{THz}$, and there is currently research on the further extension, for example into the short-band (S-band) using wavelengths below $1530 \mathrm{~nm}$ to further increase link throughputs (Fig. 1). A number of challenges need to be overcome to achieve this.
One is the development of optical amplifiers and other photonic component technologies which operate at such wavelengths. A second issue concerns the increased level of signal distortion arising from the nonlinearity of the transmission fibers.

Such technologies will be used in open optical networks, which require network planning tools to allow multi-vendor transport systems or white-box transport solutions [1-6]. Such open tools for modeling and planning optical networks must be able to take into account physical layer impairments, including fiber nonlinearity. Accurate methods to calculate nonlinearityinduced impairments are needed, and become increasingly important as signal bandwidths are extended beyond the C-band. In this paper, we study the impact of fiber nonlinearity on wideband signal transmission, review recently developed analytical models to quickly and accurately predict the amount of signal distortion and carry out a study using such an analytical model to assess throughputs.

Optical signals are distorted by a number of effects arising from the intensity-dependence of the silica, referred to as the Kerr nonlinearity: self-phase modulation (SPM, the distortion of a WDM channel due to its own intensity modulation), and the inter-channel nonlinear effects of cross-phase modulation 
(XPM), and four-wave mixing. (FWM) [7]. A further nonlinear

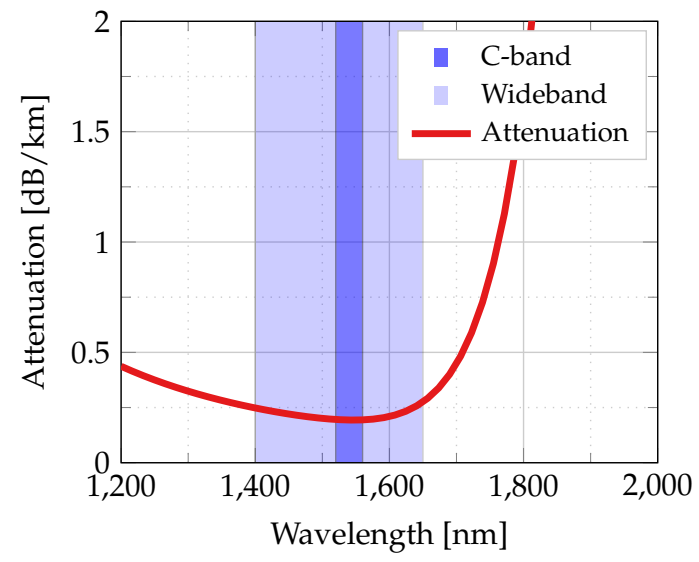

Fig. 1. Attenuation coefficient as a function of wavelength for a standard single mode fiber [Eq. (3.3) and ex. 3.3 8].

phenomenon is that of inter-channel stimulated Raman scattering, whereby inelastic scattering of the signal photons by the silica results in the generation of optical phonons and the transfer of power from higher to lower frequency WDM channels (Fig. 2) [9]. The Kerr effect arises from the quasi-instantaneous (with respect to the optical field) fiber response, whereas ISRS is a consequence of the delayed part of the nonlinear response. The power transfer due to ISRS is described by the Raman gain spectrum. In Silica, the Raman spectrum increases approximately linearly with the channel frequency separation (up to around $15 \mathrm{THz}$ ), and in a C-band system, in which the spacing between any pair of channels is $\leq 4.3 \mathrm{THz}$, power transfer due to ISRS is low and can be mitigated using gain flattening filters. However, as the WDM signal bandwidth is extended, the impact of ISRS increases and cannot be ignored when calculating system performance. It is therefore necessary to include the effect of ISRS in any model of nonlinear wideband WDM signal transmission.

\section{NONLINEAR TRANSMISSION MODEL}

The split-step Fourier method (SSFM) is a commonly-used approach to numerically solve the nonlinear Schrödinger equation (NLSE) and simulate nonlinear WDM signal transmission [7]. However, even with the use of fast computational hardware such as graphical processing units (GPUs), the simulation times can be substantial, particularly for $\mathrm{C}+\mathrm{L}$ band or wider transmission. Consequently, to speed up the process of predicting system performance, analytical models have been developed, one of the most widely-used being the Gaussian noise (GN) model of nonlinear interference (NLI) $[10,11]$, in which the NLSE is analytically solved (to first-order) using regular perturbation theory and assuming a Gaussian symbol constellation. Although symbol constellations of practical interest are not Gaussian, the accumulated uncompensated dispersion along the fiber link causes the signal in each WDM channel to evolve into a Gaussian-like waveform. This observation motivated the approximation of actual symbol constellations by a complex, circular Gaussian distribution. The aim of the GN model is to find an analytical expression of the power spectral density $G(f)$ of the first-order perturbation caused by the nonlinear term in the NLSE. The PSD of the nonlinear perturbation can then be integrated over the bandwidth of interest to obtain an equivalent AWGN power $P_{\mathrm{NLI}}=\eta_{n}\left(f_{i}\right) P_{i}^{3}$.
The GN model results in multi-dimensional (2-5 depending on, e.g, bandwidth, amplification scheme, receiver filter shape, etc.) integral expressions giving the nonlinear interference (NLI) coefficient $\eta_{n}\left(f_{i}\right)$, which is dependent on the nonlinearity coefficient, the fiber dispersion and the power profiles of the WDM channels along the link. Using those integral expressions, the NLI coefficient can be computed far more rapidly than by using SSFM-based simulation. They can also be used to obtain closed-form approximations, allowing calculations to be carried out on sub-microsecond time scales, even for long-haul wideband WDM transmission. The nonlinear interference coefficient $\eta_{n}\left(f_{i}\right)$ for the WDM channel at frequency $f_{i}$, can then be used to calculate the signal-to-noise ratio of the received WDM channel [10]

$$
\mathrm{SNR}_{i} \approx \frac{P_{i}}{P_{\mathrm{ASE}}+\eta_{n}\left(f_{i}\right) P_{i}^{3}},
$$

where $P_{i}$ is the launched signal power of the $i$ 'th WDM channel, and $P_{\text {ASE }}$ is the amplified spontaneous emission noise introduced by the in-line optical amplifiers. As both the ASE and NLI noise are modeled as Gaussian distributions with zero mean (after filtering), the total noise variance can be obtained by summing over the individual variances. Eq. (1) assumes that ASE noise and signal-signal nonlinear distortion dominate, and other sources of noise and distortion (e.g., transceiver noise, signalASE nonlinear interaction and the filtering effects of optical and electrical components) are neglected. The effect of power trans-

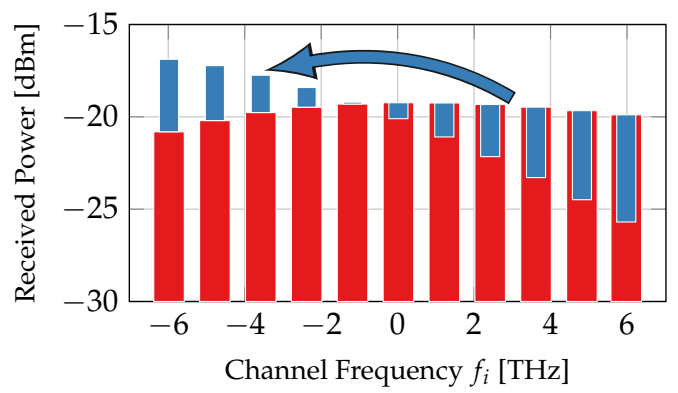

Fig. 2. An illustration of the received power without ISRS (in red) and the power transfer due to ISRS (in blue).

fer between WDM channels due to inter-channel stimulated Raman scattering can be included in the GN model of nonlinear interference. The coupling between WDM channel $i$ at frequency $f_{i}$ and all the other channels over distance $z$ along the fibre is described by [Eq. (3) 12]

$$
\frac{\partial P_{i}}{\partial z}=-\underbrace{\sum_{k=i+1}^{N_{\mathrm{ch}}} \frac{f_{i}}{f_{k}} g_{\mathrm{r}}(\Delta f) P_{k} P_{i}}_{\text {ISRS loss }}+\underbrace{\sum_{k=1}^{i-1} g_{\mathrm{r}}(\Delta f) P_{k} P_{i}}_{\text {ISRS gain }}-\alpha\left(f_{i}\right) P_{i}
$$

where $P_{i}$ and $P_{k}$ are the powers of channels $i$ and $k$, the latter being at frequency $f_{k}, N_{\mathrm{ch}}$ is the total number of channels, $\Delta f$ is the frequency spacing between the channels, $\alpha\left(f_{i}\right)$ is the fiber attenuation in the absence of ISRS and $k=1$ denotes the highest frequency channel. The first term on the right-hand side of Eq. (2) describes the transfer of power from channel $i$ into the lower frequency channels, while the second term on the righthand side accounts for the transfer of power into the channel from channels at higher frequencies. $g_{r}(f)$ is the ISRS gain spectrum which is dependent on the frequency spacing between 
the channels. $g_{r}(f)$ increases approximately linearly for $f \in$ $[0,15] \mathrm{THz}$ (Fig. 3). In the case of this linear approximation of the ISRS gain, the Raman gain spectrum can be approximated as

$$
g_{r}(\Delta f)=C_{r} \Delta f,
$$

where $C_{r}$ is the slope of the linear regression.

The signal power profiles along the fiber link obtained from Eq. (2) are then used within the GN model formalism yielding $\eta_{n}\left(f_{i}\right)$. A number of approaches can be taken to complete the calculation of the nonlinear interference coefficient, depending on the calculation speed and the accuracy required. In one approach, the effect of ISRS can be taken into account by replacing the attenuation of the fiber, $\alpha$, with an effective, channel dependent attenuation $\alpha_{\text {eff, } i}$ in the integrals (see e.g., [13]), an approach termed the effective loss method. Alternatively, accurate power profiles of each WDM channel along the fiber spans can be used within the GN model integrals calculating $\eta_{n}\left(f_{i}\right)$, which are solved numerically. A third approach follows that of the previous one, but by making some approximations, closed-form expressions giving $\eta_{n}\left(f_{i}\right)$ are derived. The latter approach allows the most rapid calculation of the nonlinear interference. Numerical solutions have been presented in [13-18], while closed-form approximations are described in [19-21]. A direct comparison can be found in [22]. It has been analytically shown that the induced nonlinear distortions are dependent on the transmitted modulation format, where significantly more complex models have been proposed to include such dependence for C-band systems (i.e., without ISRS) [23-27]. Utilizing the link function of the ISRS GN model, those modulation format aware models can be extended to account for ISRS [20, 28, 29]. A closed-form approximation, enabling real-time estimations, that accounts for the modulation format dependence has been recently reported in [20]. However, as the optimum constellation of the fiber-optic channel remains unknown, we assume Gaussian constellations for the remainder of this work.

Solving the Raman gain equations Eq. (2) gives the power profile along the span [Eq. (7) 30].

$$
P\left(z, f_{i}\right)=P\left(0, f_{i}\right) \frac{P_{\mathrm{tot}} e^{-\alpha z-P_{\mathrm{tot}} C_{\mathrm{r}} L_{\mathrm{eff}} f}}{\int G_{\mathrm{Tx}}(v) e^{-P_{\mathrm{tot}} C_{\mathrm{r}} L_{\mathrm{eff}} v} d v},
$$

where $P_{\text {tot }}$ is the total launch power of all the channels and $L_{\text {eff }}$ is the effective length of the span with

$$
L_{\mathrm{eff}}=\frac{1-\exp (-\alpha z)}{\alpha} .
$$

The result in [Eq. (7) 30] is identical with Eq. (4). However, Eq. (4) is written in terms of frequency while the result in [30] is written in terms of wavelength (resulting in a sign difference in the exponent and a different regression slope of the Raman gain function). As described in [22], Eq. (4) is used in the integral equations of the GN model to obtain an expression for $\eta_{n}\left(f_{i}\right)$ as used in Eq. (1) to calculate the SNR:

$$
\eta_{n}\left(f_{i}\right) \approx \sum_{j=1}^{n}\left[\frac{P_{i, j}}{P_{i}}\right]^{2} \cdot\left[\eta_{\mathrm{SPM}, \mathrm{j}}\left(f_{i}\right) n^{\epsilon}+\eta_{\mathrm{XPM}, \mathrm{j}}\left(f_{i}\right)\right]
$$

where $\eta_{\mathrm{SPM}}\left(f_{i}\right)$ is the contribution to the nonlinear interference coefficient from self-phase modulation and $\eta_{\mathrm{XPM}}\left(f_{i}\right)$ is the contribution from cross-phase modulation from all the other WDM channels. $P_{i}$ is the power of channel $i$ launched into the first span, and $P_{i, j}$ is the power of channel $i$ launched into the $j^{\prime}$ th span. It is assumed that only the SPM contribution accumulates

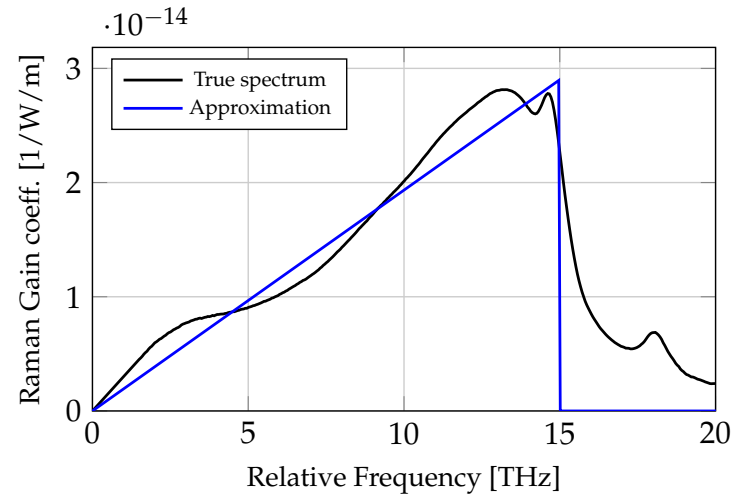

Fig. 3. Raman gain spectrum of standard SMF at $1550 \mathrm{~nm}$, and approximation with a linear regression. After [13].

coherently along the multiple fiber spans, and in Eq. (6), $\epsilon$ is the coherence factor, for which a closed form expression can be derived [11]. In the results of this work, it is assumed that the coherence factor is not a function of ISRS, which is not strictly true. However, in [17], we showed that for SMF based spans and a $10 \mathrm{THz}$ signal, neglecting the ISRS dependence on the coherence factor results in an approximation error of around 0.1 $\mathrm{dB}$ after 10 fiber spans. Additionally, formulas for the coherence factor derived in the future, that include the effect of ISRS, can simply be inserted in Eq. (6).

Closed-form approximations for $\eta_{\mathrm{SPM}}\left(f_{i}\right)$ and $\eta_{\mathrm{XPM}}\left(f_{i}\right)$ for a link with fiber attenuation $\alpha$, group velocity dispersion $\beta_{2}$, group velocity dispersion slope $\beta_{3}$, nonlinear coefficient $\gamma$, channel bandwidth $B_{i}$, are derived in [19]:

$$
\begin{aligned}
& \eta_{\mathrm{SPM}}\left(f_{i}\right) \approx \frac{4}{9} \frac{\gamma^{2}}{B_{i}^{2}} \frac{\pi}{\phi_{i} \bar{\alpha}(2 \alpha+\bar{\alpha})} \\
& .\left[\frac{T_{i}-\alpha^{2}}{\alpha} \operatorname{asinh}\left(\frac{\phi_{i} B_{i}^{2}}{\pi a}\right)+\frac{A^{2}-T_{i}}{A} \operatorname{asinh}\left(\frac{\phi_{i} B_{i}^{2}}{\pi A}\right)\right],
\end{aligned}
$$

with $\phi_{i}=\frac{3}{2} \pi^{2}\left(\beta_{2}+2 \pi \beta_{3} f_{i}\right), \quad A=\alpha+\bar{\alpha}, T_{i}=$ $\left(\alpha+\bar{\alpha}-P_{\text {tot }} C_{\mathrm{r}} f_{i}\right)^{2}$ and

$$
\begin{aligned}
& \eta_{\mathrm{XPM}}\left(f_{i}\right) \approx \frac{32}{27} \sum_{k=1, k \neq i}^{N_{\mathrm{ch}}}\left(\frac{P_{k}}{P_{i}}\right)^{2} \frac{\gamma^{2}}{B_{k} \phi_{i, k} \bar{\alpha}(2 \alpha+\bar{\alpha})} \\
& \cdot\left[\frac{T_{k}-\alpha^{2}}{\alpha} \operatorname{atan}\left(\frac{\phi_{i, k} B_{i}}{\alpha}\right)+\frac{A^{2}-T_{k}}{A} \operatorname{atan}\left(\frac{\phi_{i, k} B_{i}}{A}\right)\right],
\end{aligned}
$$

with $\phi_{i, k}=2 \pi^{2}\left(f_{k}-f_{i}\right)\left[\beta_{2}+\pi \beta_{3}\left(f_{i}+f_{k}\right)\right]$. Eq. (7) and Eq. (8) are able to account for arbitrary launch power distributions. In their derivation, ISRS is modeled to first-order which neglects the impact of non-flat launch powers on the denominator in Eq. (4). As shown in [Sec. E 17], a launch power slope of 3 $\mathrm{dB}$ imposes an approximation error of around $0.3 \mathrm{~dB}$ in NLI for the SPM contribution. However, the approximation error on the total NLI will be smaller due to additional XPM terms. Additionally, at optimum launch power, this approximation error further reduces to around $0.1 \mathrm{~dB}$ as the ASE noise is twice as large as the NLI contribution. We, therefore, believe that this error can be deemed negligible. In principle, this approximation error could be further reduced by using the parameter $\bar{\alpha}$. The parameter can be used to allow general cases, including nonuniform (e.g., tilted) launch power distributions across the WDM 
signal spectrum, wavelength-dependent attenuation and the extension of the formula for signal bandwidths beyond $15 \mathrm{THz}$. However, as the approximation error in this work is negligible, we set $\bar{\alpha}=\alpha$.

While such closed-form approximations allow very rapid calculation of the performance of long-haul wideband WDM signal transmission, their accuracy needed to be verified. We have investigated the accuracy and speed of calculations using the different models to calculate the nonlinear interference coefficient, and compared the results with the values obtained using the split-step Fourier method. In this assessment, the propagation over $6 \times 100 \mathrm{~km}$ of standard single mode fiber of $251 \times 40$ GBd channels (Nyquist spacing) with a total optical bandwidth of $10 \mathrm{THz}$, Gaussian modulation, and $0 \mathrm{dBm}$ launch power was modeled. Noise-free amplification was assumed. Using a single GPU and the split-step Fourier method, including the accurate signal power profiles along the link due to ISRS and fiber attenuation, calculation of the received signal SNRs for all 251 channels, averaging 4 data realizations, took 186 hours. By comparison, using the GN model and solving the integrals numerically, but neglecting the effect of ISRS, the calculation was completed in just 0.48 hours using a single CPU core. However, neglecting ISRS resulted in inaccuracies in the nonlinear interference of up to $2 \mathrm{~dB}$. Using the GN model but including the effective loss approximation to take into account the effect of ISRS resulted in the errors being reduced to, at most, $0.2 \mathrm{~dB}$, and a slight increase in the calculation time to 0.56 hours. Numerical calculations of the integrals, using the accurate signal power profiles, reduced the inaccuracy further to a maximum error of just $0.1 \mathrm{~dB}$, and increased computation time to 3.4 hours. It should be noted that the computation times are only given to compare orders of magnitude, as they are strongly dependent on implementation, simulation parameters and available hardware. It should also be noted that the phase-array factor was included in the ISRS GN model, to account for coherent NLI accumulation, which is known to impose significant computational complexity. Finally, the closed-form expressions Eq. (7) and Eq. (8) allowed the calculation to be performed in less than $1 \mathrm{~s}$, with a maximum error in the calculated received signal SNR across the whole WDM spectrum of $0.3 \mathrm{~dB}$ (Fig. 4). We conclude that, in many applications, the closed-form approximation offers an excellent compromise between speed and accuracy. We developed the code in [31], that has been used to obtain the results in this work.

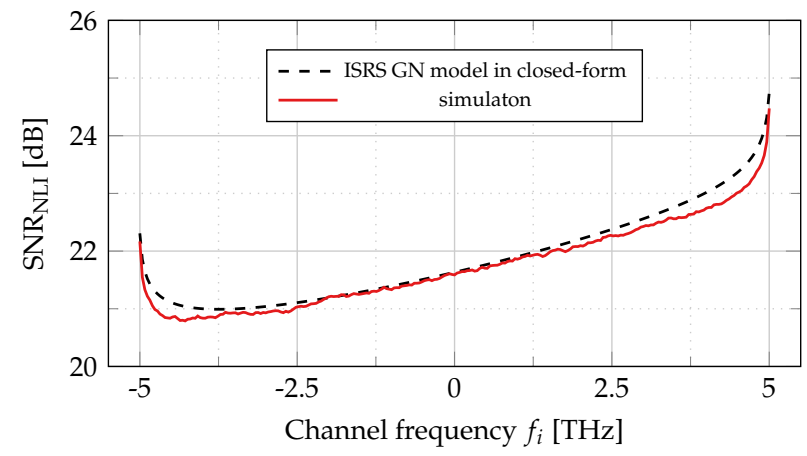

Fig. 4. Signal-to-noise ratios in $6 \times 100 \mathrm{~km}$ link, including Kerr nonlinearity and ISRS, calculated using the split-step Fourier method (dashed line) and closed-form expressions (5)-(7). After [19].

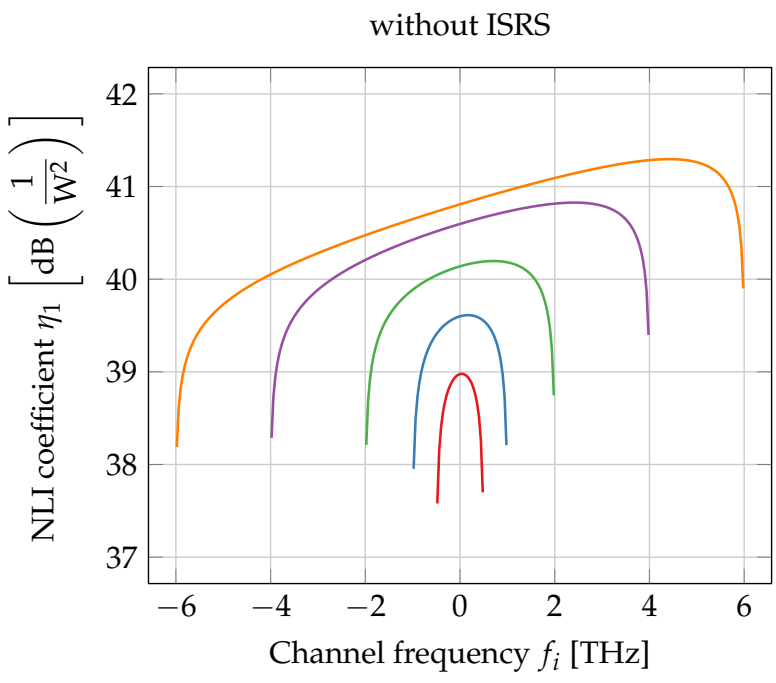

with ISRS

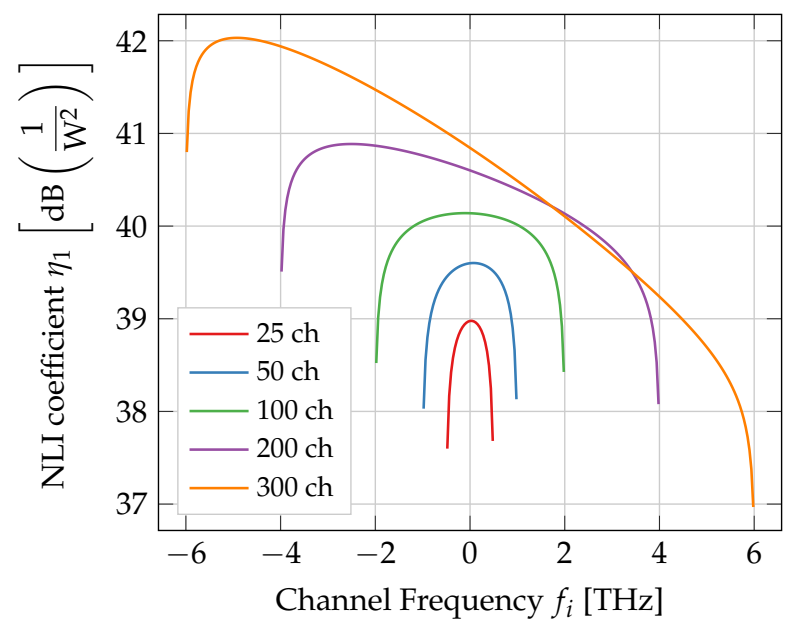

Fig. 5. Nonlinear interference coefficient calculated for WDM transmission over $1000 \mathrm{~km}$ with signal bandwidths of 1,2, 4, 8 and 12 THz. Top: Neglecting the effect of ISRS. Bottom: Including the effect of ISRS.

\section{CALCULATIONS OF ACHIEVABLE INFORMATION RATES IN WIDEBAND WDM SIGNAL TRANSMISSION}

The ISRS GN model described in the previous section can be used to estimate the achievable information rates (AIR) of wideband WDM systems, assisting telecom network operators aiming to fully exploit their existing fiber infrastructure to optimize their returns on CAPEX. In open optical networks, the use of simple, fast and accurate transmission models will be utilized in software-defined networking (SDN) solutions, to select optimum lightpath routes and predict data throughputs. In this section, achievable information rates in wideband WDM links, rapidly calculated using the closed-form approximations, are presented.

We assessed WDM transmission systems, centered at wavelength $1550 \mathrm{~nm}$ with Nyquist-spaced 40 Gbaud channels, standard single mode fiber with dispersion of $D=17 \mathrm{ps} / \mathrm{nm} / \mathrm{km}$ $\left(\beta_{2}=-21.7 \mathrm{ps}^{2} / \mathrm{km}\right)$, dispersion slope $S=0.067 \mathrm{ps} / \mathrm{nm}^{2} / \mathrm{km}$ $\left(\beta_{3}=0.144 \mathrm{ps}^{3} / \mathrm{km}\right)$, nonlinear coefficient $\gamma=1.21 / \mathrm{W} / \mathrm{km}$, ISRS gain slope $C_{r}=0.0281 / \mathrm{W} / \mathrm{km} / \mathrm{THz}$ at $1550 \mathrm{~nm}$ and 


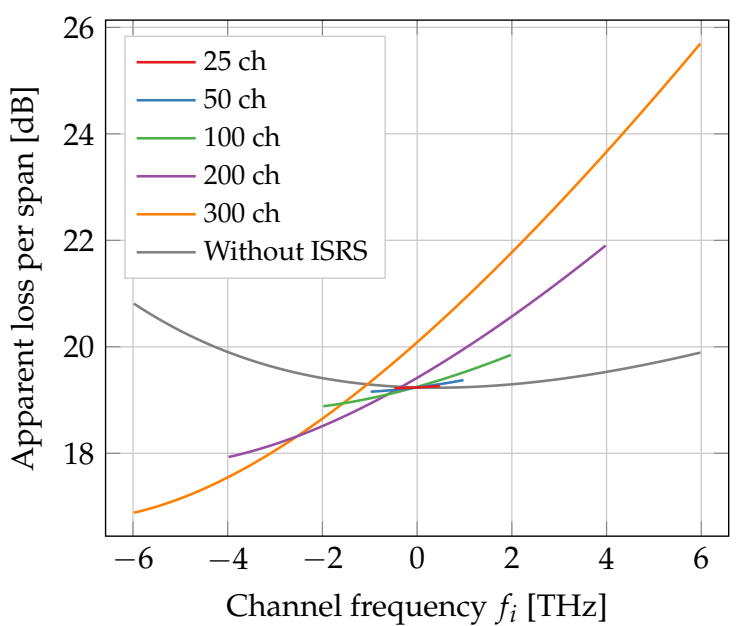

Fig. 6. Apparent span loss as a function of channel frequency with $0 \mathrm{dBm}$ per channel launch power, for signal bandwidths of $1,2,4,8$ and $12 \mathrm{THz}$.

$10 \times 100 \mathrm{~km}$ spans for a transmission distance of $1000 \mathrm{~km}$. Inline lumped amplifiers with a noise figure $\mathrm{NF}=4.5 \mathrm{~dB}$, were assumed, with a spectral shaping filter (dynamic spectral equalizer) at each amplifier being employed to achieve uniform signal launch power across the spectral band into each span of $0 \mathrm{dBm}$ per channel. Signal bandwidths ranging from $1 \mathrm{THz}$ to $12 \mathrm{THz}$ (channel counts ranging from 25 to 300 channels) were considered. Fig. 5 shows the nonlinear interference coefficient obtained from closed-form expressions Eq. (7) and Eq. (8), firstly with the effect of ISRS neglected (Fig. 5 top), and then with ISRS included (Fig. 5 bottom). In the case of the signal with a bandwidth of $12 \mathrm{THz}$, with the effect of ISRS neglected, a maximum value of $\eta_{n}\left(f_{i}\right)=41.4 \mathrm{~dB}\left(1 / \mathrm{W}^{2}\right)$ at a frequency of $+4.8 \mathrm{THz}$ (relative to the center frequency) is observed. There is a pronounced spectral tilt in $\eta_{n}\left(f_{i}\right)$, with higher frequency channels experiencing higher levels of nonlinear distortion, which can be explained by the fiber's dispersion slope. The lower dispersion at the higher frequencies leads to reduced walk-off between spectral components at the higher frequencies, and hence increased nonlinear distortion. The results of the calculations of $\eta_{n}\left(f_{i}\right)$ including ISRS (Fig. 5 bottom) shows that the main effect of inter-channel stimulated Raman scattering is to reverse the spectral tilt of the nonlinear interference coefficients with higher $\eta_{n}\left(f_{i}\right)$ at the lower frequencies, caused by ISRS transferring power into the lower frequency channels, which consequently experience increased distortion due to SPM and XPM.

The values of the channel launch powers $P_{i}$, amplified spontaneous emission from the in-line amplifiers, $P_{\mathrm{ASE}}$, and the nonlinear interference coefficients $\left(\eta_{n}\left(f_{i}\right)\right)$ can be used in Eq. (1) to calculate the received signal $\mathrm{SNR}_{i}$, from which the achievable information rate (AIR) of each WDM channel, in bits/symbol, can be calculated [p. 45 32]

$$
\mathrm{AIR}_{i}=2 \log _{2}\left(1+\mathrm{SNR}_{i}\right)
$$

where the factor of 2 on the right-hand side arises from the two polarizations. The ASE noise power spectral density from each amplifier at the frequency of the $i^{\prime}$ th channel is [33]

$$
P_{\mathrm{ASE}, i}=2\left(G_{i}-1\right) n_{\mathrm{sp}} h\left(f_{c}+f_{i}\right) B_{i}
$$

where $n_{\mathrm{sp}} \approx \frac{\mathrm{NF}}{2}$ is the spontaneous emission factor, $h$ Planck's constant, $f_{c}$ is the reference frequency and $G_{i}=P_{i}(L) / P_{i}(0)$ is

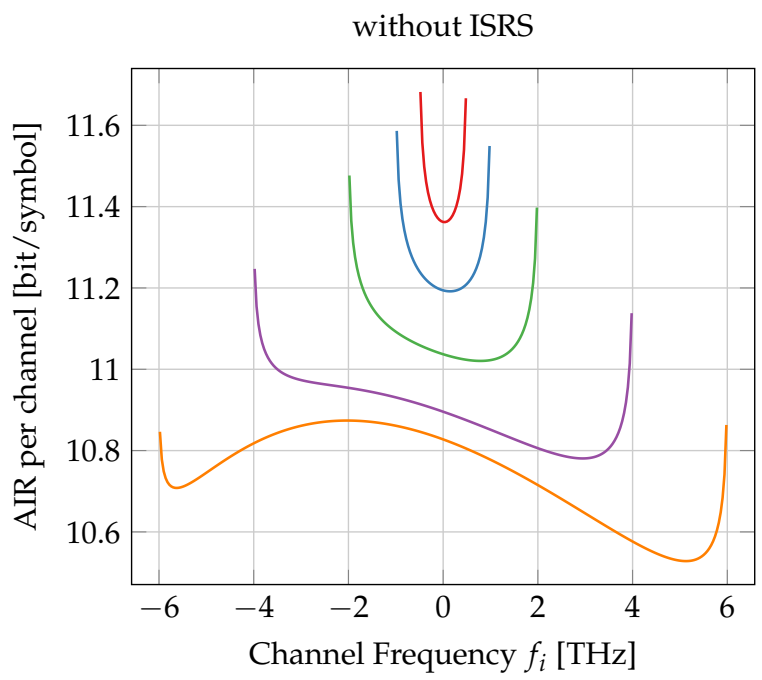

with ISRS

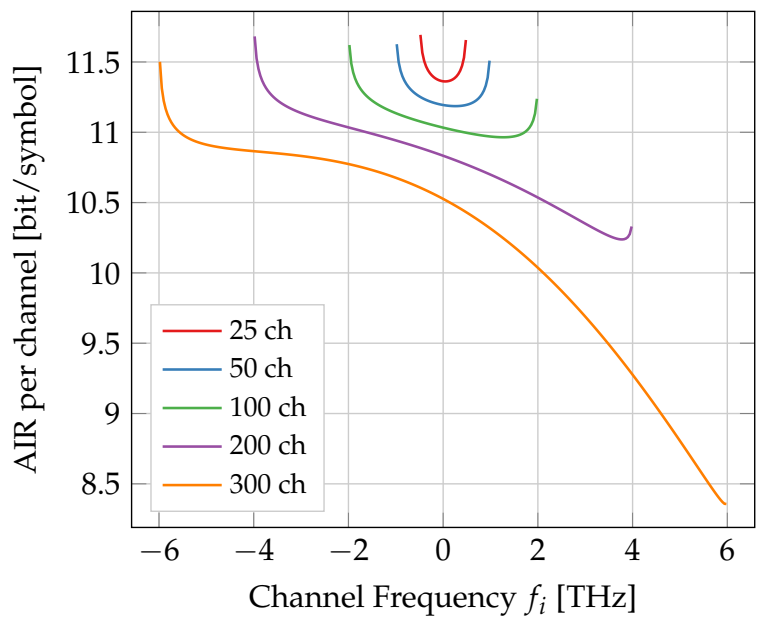

Fig. 7. Achievable information rates per dual polarisation channel calculated for WDM transmission over $1000 \mathrm{~km}$ with signal bandwidths of 1, 2, 4, 8 and $12 \mathrm{THz}$. Top: Neglecting the effect of ISRS. Bottom: Including the effect of ISRS.

the amplifier gain at the frequency of the $i^{\prime}$ th channel, where $P_{i}(0)$ and $P_{i}(L)$ are the powers of channel $i$ at the input and output of the span, respectively, given by Eq. (4) with $z=L$.

Fig. 6 shows the apparent span loss, as a function of channel frequency, for systems with WDM signal bandwidths from 1 to $12 \mathrm{THz}$ and $0 \mathrm{dBm}$ per channel launch power. The impact of ISRS can be seen in the increasing slope of the loss with frequency.

With ISRS, increasing the signal bandwidth from 1 to $12 \mathrm{THz}$ causes a reduction in the minimum AIR of the worst performing channel from 11.3 bits/symbol to 8.4 bits/symbol (Fig. 7). At the signal bandwidth of $12 \mathrm{THz}$, a large variation in the achievable information rates across the WDM band is observed, both in the case without and that with ISRS. However, the variation of the AIR across the channels becomes significantly larger in the presence of ISRS. The AIR of the worst performing channel falls from 10.5 bits/symbol in the absence of ISRS to just 8.4 bits/symbol in the presence of ISRS.

Although the value of the nonlinear interference coefficient, is lower for the higher frequency channels (Fig. 5), the high 
apparent span losses resulting from the ISRS lead to lower SNR. It should be noted that these rates represent an upper bound on the rates that can be achieved in practice. To fully exploit the fiber infrastructure and operate as close to capacity as possible, coded modulation would be used, with constellation shaping and soft-decision forward error correction. The values of $\eta_{n}\left(f_{i}\right)$ calculated in this study make the assumption that the signals have Gaussian distributions. In the case of constellation shaping, this assumption may be valid. However, to obtain more accurate results, a modulation format correction formula should be used in the ISRS GN model [20].

\section{OPTIMIZATION OF LAUNCH POWER AND GAIN EQUALIZATION}

In the previous sections, we have described a model of nonlinear signal distortion in wideband optical fiber transmission, including the effect of inter-channel stimulated Raman scattering. We showed results validating the model's accuracy by comparison with split-step Fourier method simulations, and studied the impact of ISRS on the achievable information rate per channel in wideband transmission systems. A key problem to be solved using open optical network planning tools concerns the design and planning of links and networks to obtain a good balance between high total throughput, and low cost and complexity of the link.

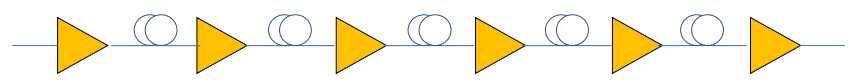

(a)

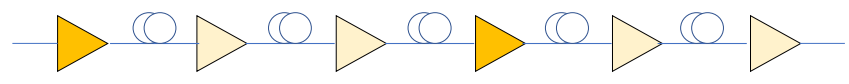

(b)

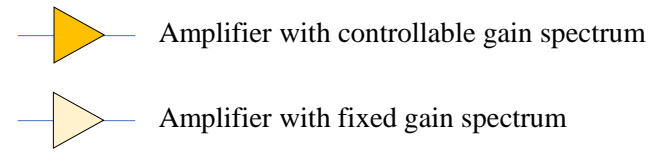

Fig. 8. Schematics of links using (a) amplifiers with dynamic gain equalization in every span, (b) both amplifiers with dynamic gain equalization and uniform gain spectra.

In the links considered in the previous section, the launch powers per channel were assumed to be uniform across the signal spectrum, with each inline amplifier having dynamic gain equalization. However, the use of uniform launch power leads to variations in received signal SNR across the spectrum, and is not necessarily optimum in terms of maximizing throughput. In addition, the use of dynamic gain equalization at each amplifier to maintain the uniform launch power per channel into each span may lead to high CAPEX for the link.

In this section, we investigate the use of optimized, nonuniform launch power spectral profiles and in-line optical amplifiers with both dynamic gain equalization and fixed, uniform gain spectra. Such studies using the ISRS GN model can be used in network planning tools to achieve the best compromises between performance and cost. Optimizing the channel launch power to maximize the minimum channel margin has been investigated in [15]. As the optimization problem is not convex, the authors propose a convex approximation of the optimization
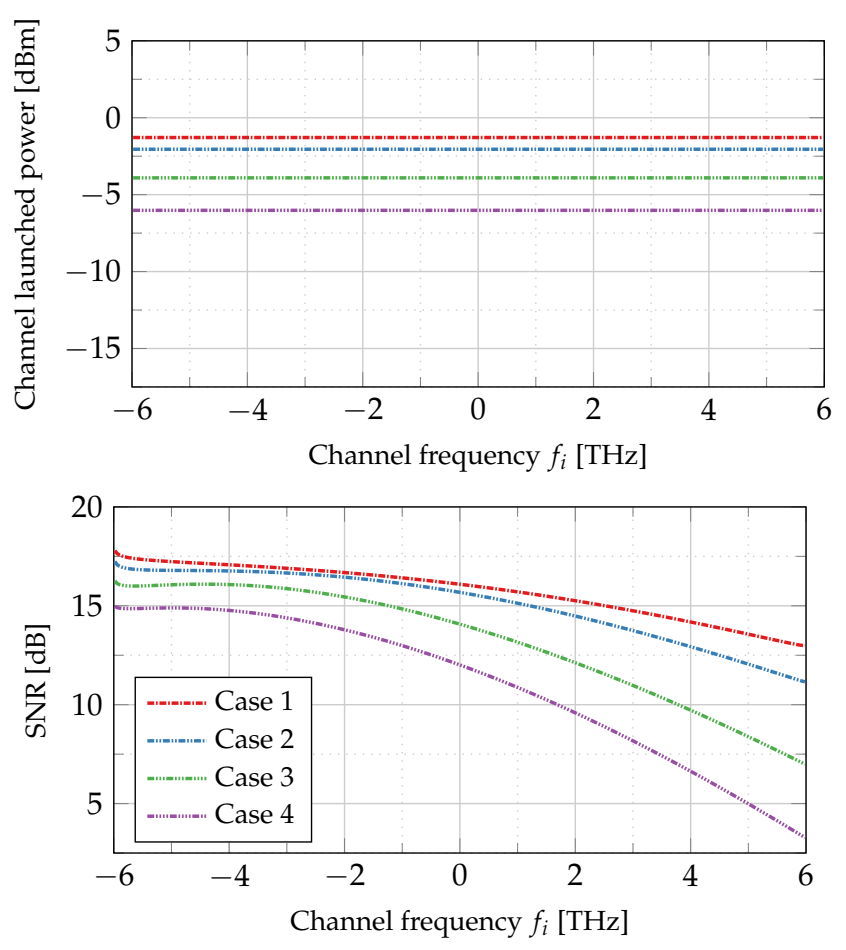

Fig. 9. $1000 \mathrm{~km}$ transmission system modeling results for Cases 1-4 with spectrally-uniform launch powers. The total achievable information rates are 126.6, 121.4, 107.2 and 91.5 $\mathrm{Tb} / \mathrm{s}$, respectively. Top: Launch power per channel for each case. Bottom: SNR values per channel.

problem and alternatively freeze the impact of ISRS and NLI on the SNR to achieve convergence. In [34], the total AIR is maximized by considering a flat launch power over respective transmission bands (i.e., flat launch power over C and L-band respectively) to significantly reduce the optimization space, but yielding a sub-optimal solution as launch powers are not optimized individually. In this section a different approach is pursued. Instead of approximating the mathematical description of the optimization problem or reducing the optimization space, we use the closed-form approximation of the ISRS GN model, approximating only the NLI contribution with negligible errors as discussed in Section 2. The use of closed-form expressions yields vast reductions in execution time of individual function calls avoiding the necessity of approximations of the optimization problem or reductions of the optimization space. Arbitrary combinations of the approaches are, of course, possible.

Fig. 8 illustrates the links being considered. In link (a), every in-line amplifier has dynamic gain equalization, allowing the launch powers of the individual channels to be optimized for every span. While this may offer the highest total throughput, the CAPEX is high, and cost-savings can be made by using a lower number of dynamic gain equalizing amplifiers deployed infrequently along the link, interspersed by amplifiers with fixed, uniform gain (link (b)). 

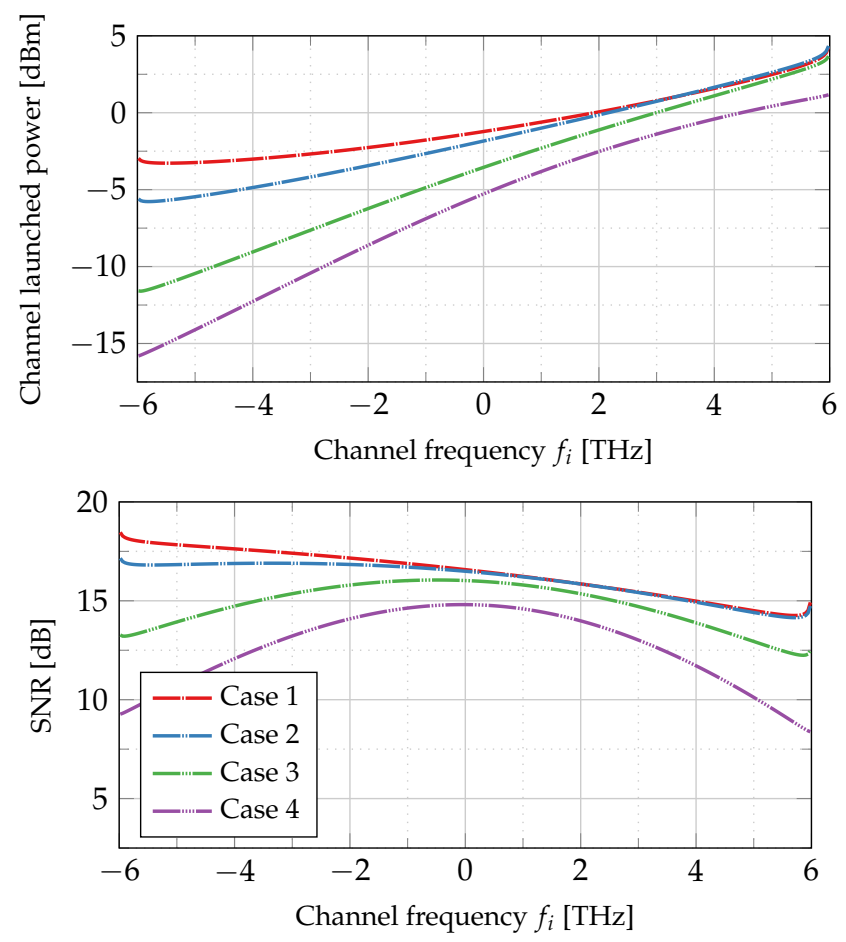

Fig. 10. $1000 \mathrm{~km}$ transmission system modeling results for Cases 1-4 with optimized launch powers. The total achievable information rates are 131.5, 129.1, 119.0 and $103.0 \mathrm{~Tb} / \mathrm{s}$, respectively. Top: Launch power per channel for each case. Bottom: SNR values per channel.

Four cases were considered, each with a total transmission distance of 10 spans $(1000 \mathrm{~km})$ :

Case 1: Every amplifier recovers the transmitted launch power Case 2: Every second amplifier recovers the transmitted launch power

Case 3: Every fifth amplifier recovers the transmitted launch power

Case 4: No amplifiers recover the transmitted launch power

All other link parameters were kept the same as in the study described in Section 3. Initially, the performance for the above four cases was calculated assuming uniform channel launch powers. The launch power was optimized to achieve the highest average AIR. Although the total AIR has been shown to be convex with respect to logarithmic launch powers in the absence of ISRS, launch power optimization in the presence of ISRS is a nonconvex optimization problem [15]. For a good balance between local and global search, the optimization was carried out using particle swarm optimization (PSO) and a subsequent steepest descent algorithm. The steepest descent algorithm requires $N_{\mathrm{ch}}$ function evaluations per optimization step to numerically calculate the gradient of the objective function. This can be done within micro-seconds due to the closed-form approximations. However, the computational complexity could be even further reduced by analytically deriving the gradient of the objective functions Eq. (11) and Eq. (12). The steepest descent algorithm is efficient in converging to a local minimum given a good initial solution, however it may get stuck in sub-optimal local minima or saddle points. The overcome this problem, the initial condition of the steepest descent algorithm is determined by the PSO. While the PSO is very inefficient in finding exact minima, it has good exploration properties. The optimization was carried out
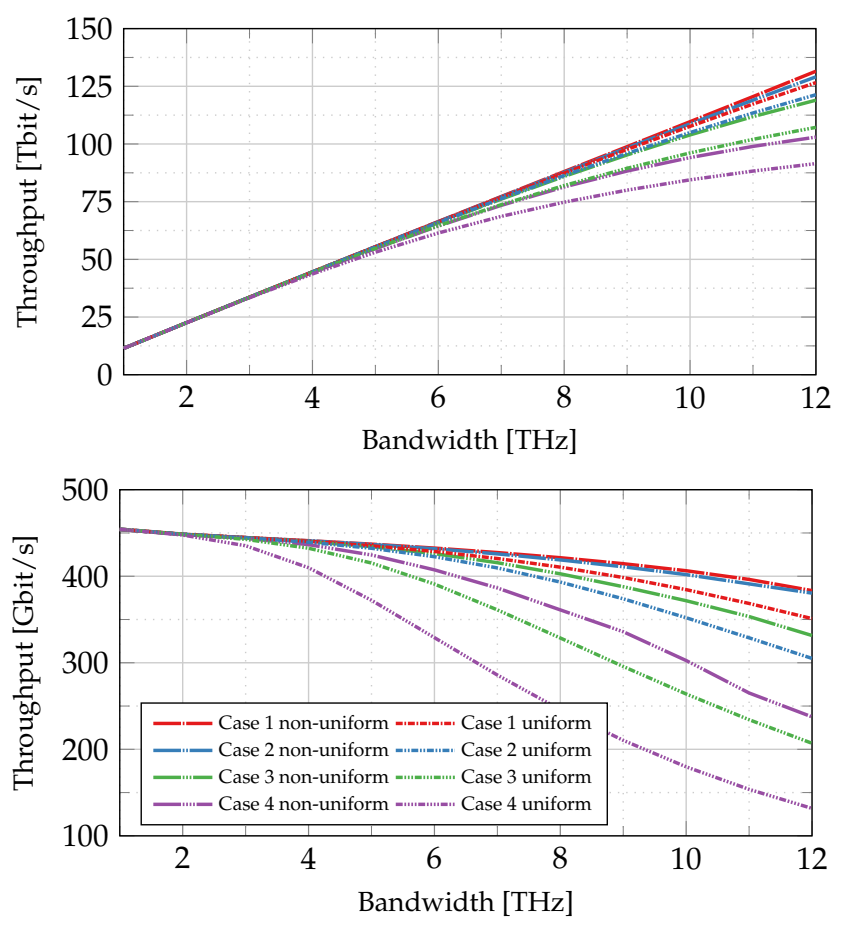

Fig. 11. $1000 \mathrm{~km}$ transmission system modeling results, with uniform and non-uniform spectral power profiles. Top: Total link throughput. Bottom: Throughput of the worst-performing channel in each case.

in logarithmic coordinates to have better curvature properties $[35,36]$. In the PSO, the population size was $10 \cdot N_{\mathrm{ch}}$, initial launch powers were picked randomly within $[-30,15] \mathrm{dBm}$, initial particle velocities were randomly picked between $[-45,45]$ and the initial inertia was 1.1. For the steepest descent the stopping criterium for the first-order optimality was $10^{-3}$ with a line search to determine the step size according to [Sec. 2-6 37].The unconstrained optimization problems, that maximize the total (and average per channel) AIR, can be mathematically described as

$$
P_{\mathrm{opt}, i}=\underset{P_{i}}{\arg \min } \sum_{\forall i}-\log _{2}\left(1+\frac{P_{i}}{P_{\mathrm{ASE}}\left(f_{i}\right)+\eta_{n}\left(f_{i}\right) P_{i}^{3}}\right),
$$

with launch power vector $P_{i}$ for a non-flat launch power distribution and

$$
P_{\text {opt }, \text { flat }}=\underset{P_{i}}{\arg \min } \sum_{P_{\text {flat }}}-\log _{2}\left(1+\frac{P_{\text {flat }}}{P_{\text {ASE }}\left(f_{i}\right)+\eta_{n}\left(f_{i}\right) P_{\text {flat }}^{3}}\right)
$$

where for all channel launch powers $P_{i}=P_{\mathrm{opt}, \text { flat }} \forall i$ for a flat launch power distribution. Amplifiers with dynamic gain equalization are then set by using Eq. (6). Using standard MATLAB implementations, the computation times ranged from seconds to minutes depending on accuracy requirements and when the best solution of the PSO was passed to the steepest descent method. The computation speed could be further improved by lumping adjacent channels into super channels, as adjacent channels are likely to exhibit similar launch powers. Fig. 9 shows the resulting optimized launch powers, and the received SNR per channel. In Case 1, with dynamic gain equalization in every span, the optimum launch power was $-1 \mathrm{dBm}$ per channel, and the SNR 
varied between 13 and $17 \mathrm{~dB}$, with the highest channels experiencing the lowest SNRs, due to ISRS. In Case 2, with dynamic gain equalization in every second span, the optimum launch power dropped to $-2 \mathrm{dBm}$, and the variation in received SNRs increased to $6 \mathrm{~dB}$ (between 12 and $17 \mathrm{~dB}$ ). The trend continues as the spacing between the equalizers increases; in the extreme case of the link without dynamic gain equalization, the optimum launch power was $-6 \mathrm{dBm}$, and a significant impact on performance is seen, with received SNRs of between $3 \mathrm{~dB}$ and $15 \mathrm{~dB}$.

Next, the study was repeated, but with optimized, nonuniform channel powers across the signal spectrum. Fig. 10 shows the resulting optimized launch powers, and the received SNR per channel. In Case 1, with dynamic gain equalization in every span, the optimum launch power ranged between -3 and $+4 \mathrm{dBm}$ across the $15 \mathrm{THz}$ signal band (the higher launch powers being used for the higher frequency channels to offset the additional link loss arising from ISRS). The SNR varied between 14 and $19 \mathrm{~dB}$, with the highest channels experiencing the lowest SNRs, due to ISRS. This is an approximately $1 \mathrm{~dB}$ increase across the signal spectrum compared with the case with uniform launch powers. In Case 2, with dynamic gain equalization in every second span, the launch power tilt increased, compensating for the additional impact of ISRS, and the variation in received SNRs increased to $3 \mathrm{~dB}$ (between 14 and $17 \mathrm{~dB}$ ), with an approximately $1 \mathrm{~dB}$ higher SNR across the signal spectrum compared with the case with uniform launch powers. The trend continues as the spacing between the equalizers increases; in the extreme case of the link without dynamic gain equalization, the received SNRs of between $8 \mathrm{~dB}$ and $15 \mathrm{~dB}$ represent a significant improvement compared to the 3 to $15 \mathrm{~dB}$ range obtained with uniform launch powers.

Besides assessing the variation in received signal SNRs across the signal band for the different link designs and launch power strategies, it is also of interest to assess the achievable information rates, both for the worst-performing channel and for the link as a whole. These results are plotted in Fig. 11, for all four cases, and for both uniform and non-uniform launch powers as a function of signal bandwidth. The presence of ISRS results in the throughput of wideband systems, i.e., those with bandwidths beyond $5 \mathrm{THz}$ bandwidth, becoming more dependent on optimization of channel powers and the spacing of dynamic gain equalization along the link. In the case of $12 \mathrm{THz}$ bandwidth transmission, the total throughput varies from the lowest value of $90 \mathrm{~Tb} / \mathrm{s}$ with uniform channel powers and no in-line dynamic gain equalization, to $130 \mathrm{~Tb} / \mathrm{s}$ with optimized nonuniform launch powers and dynamic gain equalizers at every span.

From the results of this study, the following conclusions can be drawn: launch power optimization results in increased uniformity of the received SNR across the wavelengths. When no launch power optimization is performed, a few channels suffer from a very low SNR. A more uniform SNR can also be achieved by introducing more closely-spaced adaptive gain equalizers. The overall gain of the total throughput using launch power optimization can be around $10 \%$, although the gain from launch power optimization is reduced when more frequent gain equalization is employed.

The application of the ISRS GN model in studies such as that described in this section can provide a powerful technique in network planning tools for open optical networks, for link design, routing, spectrum and power assignment in open optical networks, and for physical-layer-aware software-defined network control and management

\section{CONCLUSIONS}

Wideband WDM technologies allow increased fiber link and network capacities, and may be attractive for telecom network operators aiming to fully exploit their existing fiber infrastructure to optimize their returns on CAPEX. Wideband WDM is also compatible with space division multiplexing, offering a path to future link capacities in the petabit/s range. Computer models of such systems are required which allow maximum data rates to be rapidly and accurately calculated, for a given transmission distance and fiber and amplifier characteristics. Such models will be vital in the operation of open optical networks to allow channel parameters and achievable information rates to be accurately predicted, and will be utilized in software-defined networking (SDN).

In this paper, we described the nonlinear effects observed in wideband transmission systems, including inter-channel stimulated Raman scattering (ISRS), and reviewed recently-developed analytical tools, based on the Gaussian noise model of nonlinear interference, incorporating the effect of ISRS. Using the ISRS GN model, we carried out a study assessing the impact of fiber nonlinearity on the achievable information rates in $1000 \mathrm{~km}$ fiber transmission systems with bandwidths of up to $12 \mathrm{THz}$, quantifying the impact of the fiber nonlinearity on the AIR, and, in particular, focusing on the effect of ISRS on the distribution of achievable information rates across the WDM band.

We demonstrated the use of the model in the optimization of launch power spectral profiles for a variety of dynamic gain equalizer arrangements in a $1000 \mathrm{~km}$ standard SMF link, using particle swarm optimization and the steepest descent algorithm. Such nonlinear models and optimization methods could be applied in OON planning tools, for example in optical link emulators to estimate quality-of-transmission (QoT) and link throughput, and in impairment-aware software-defined network control and management.

\section{FUNDING}

Financial support from UK EPSRC through programme grant TRANSNET (EP/R035342/1) is gratefully acknowledged.

\section{ACKNOWLEDGMENTS}

The authors would like to thank the anonymous reviewers for their feedback that helped improve this manuscript.

\section{REFERENCES}

1. B. D. Taylor, G. Goldfarb, S. Bandyopadhyay, V. Curri, and H. Schmidtke, "Towards a route planning tool for open optical networks in the telecom infrastructure project," in 2018 Optical Fiber Communications Conference and Exposition (OFC), (2018), pp. 1-3.

2. "https://telecominfraproject.com/oopt/," accesses: 13/02/2020 .

3. M. Filer, M. Cantono, A. Ferrari, G. Grammel, G. Galimberti, and V. Curri, "Multi-vendor experimental validation of an open source qot estimator for optical networks," J. Light. Technol. 36, 3073-3082 (2018).

4. J. Auge, G. Grammel, E. le Rouzic, V. Curri, G. Galimberti, and J. Powell, "Open optical network planning demonstration," in 2019 Optical Fiber Communications Conference and Exhibition (OFC), (2019), pp. 1-3.

5. L. Alahdab, E. Le Rouzic, J. Meuric, J. Augé, C. Ware, and K. Ndiaye, "Demonstration of a multivendor path computation with optical feasibility combining gmpls and open source," in 2019 Optical Fiber Communications Conference and Exhibition (OFC), (2019), pp. 1-3. 
6. J. Auge, V. Curri, and E. Le Rouzic, "Open design for multi-vendor optical networks," in 2019 Optical Fiber Communications Conference and Exhibition (OFC), (2019), pp. 1-3.

7. G. Agrawal, Nonlinear Fiber Optics (Elsevier Science Publishing Co Inc, 2012).

8. G. Keiser, Optical fiber communications (Wiley Encyclopedia of Telecommunications, 2010).

9. $\quad$ R. H. Stolen and E. P. Ippen, "Raman gain in glass optical waveguides," App. Phys. Lett. 22, 276-278 (1973)

10. A. Splett, C. Kurtzke, and K. Petermann, "Ultimate transmission capacity of amplified optical fiber communication systems taking into account fiber nonlinearities," in 1993 The European Conference on Optical Communication (ECOC), (1993).

11. P. Poggiolini, "The GN model of non-linear propagation in uncompensated coherent optical systems," J. Light. Technol. 30, 3857-3879 (2012).

12. S. Tariq and J. C. Palais, "A computer model of non-dispersion-limited stimulated Raman scattering in optical fiber multiple-channel communications," J. Light. Technol. 11, 1914-1924 (1993).

13. D. Semrau, R. Killey, and P. Bayvel, "Achievable rate degradation of ultra-wideband coherent fiber communication systems due to stimulated Raman scattering," Opt. Express 25, 13024-13034 (2017).

14. M. Cantono, D. Pilori, A. Ferrari, and V. Curri, "Introducing the generalized $\mathrm{GN}$-model for nonlinear interference generation including space/frequency variations of loss/gain," ArXiv e-prints (2017).

15. I. Roberts, J. M. Kahn, J. Harley, and D. W. Boertjes, "Channel power optimization of WDM systems following Gaussian noise nonlinearity model in presence of stimulated Raman scattering," J. Light. Technol. 35, 5237-5249 (2017).

16. M. Cantono, J. L. Auge, and V. Curri, "Modelling the impact of SRS on $\mathrm{NLI}$ generation in commercial equipment: an experimental investigation," in Optical Fiber Communication Conference, (Optical Society of America, 2018), p. M1D.2.

17. D. Semrau, R. I. Killey, and P. Bayvel, "The Gaussian Noise model in the presence of inter-channel stimulated Raman scattering," J. Light. Technol. 36, 3046-3055 (2018).

18. D. Semrau, E. Sillekens, R. I. Killey, and P. Bayvel, "The ISRS GN model, an efficient tool in modeling ultra-wideband transmission in point-to-point and network scenarios," in 2018 European Conference on Optical Communication (ECOC), Tu4G.6, (2018), pp. 1-3, pre-print available in arxiv:1808.00533.

19. D. Semrau, R. I. Killey, and P. Bayvel, "A closed-form approximation of the Gaussian noise model in the presence of inter-channel stimulated Raman scattering," J. Light. Technol. pp. 1-1 (2019).

20. D. Semrau, E. Sillekens, R. I. Killey, and P. Bayvel, "A modulation format correction formula for the Gaussian noise model in the presence of inter-channel stimulated Raman scattering," J. Light. Technol. 37, 5122-5131 (2019).

21. P. Poggiolini, "A generalized GN-model closed-form formula," arXiv:181006545v2 (Sep. 2018)

22. D. Semrau, R. I. Killey, and P. Bayvel, "Overview and comparison of nonlinear interference modelling approaches in ultra-wideband optical transmission systems," Int. Conf. on Transparent Opt. Networks (ICTON) (2019)

23. R. Dar, M. Feder, A. Mecozzi, and M. Shtaif, "Properties of nonlinear noise in long, dispersion-uncompensated fiber links," Opt. Express 21, 25685 (2013)

24. R. Dar, M. Feder, A. Mecozzi, and M. Shtaif, "Accumulation of nonlinear interference noise in fiber-optic systems," Opt. Express 22, 1419914211 (2014).

25. A. Carena, G. Bosco, V. Curri, Y. Jiang, P. Poggiolini, and F. Forghieri, "EGN model of non-linear fiber propagation," Opt. Express 22, 16335 (2014).

26. A. Mecozzi and R.-J. Essiambre, "Nonlinear Shannon limit in pseudolinear coherent systems," J. Light. Technol. 30, 2011-2024 (2012).

27. M. Secondini and E. Forestieri, "Analytical fiber-optic channel model in the presence of cross-phase modulation," IEEE Photon. Technol. Lett. 24, 2016-2019 (2012).
28. C. Lasagni, P. Serena, and A. Bononi, "A raman-aware enhanced gnmodel to estimate the modulation format dependence of the snr tilt in c+l band," in European Conference on Optical Communication (ECOC), (2019), W.1.D.

29. H. Rabbani, G. Liga, V. Oliari, L. Beygi, E. Agrell, M. Karlsson, and A. Alvarado, "A general analytical model of nonlinear fiber propagation in the presence of kerr nonlinearity and stimulated raman scattering," arXiv preprint arXiv:1909.08714 (2019).

30. M. Zirngibl, "Analytical model of Raman gain effects in massive wavelength division multiplexed transmission systems," Electron. Lett. 34, 789-790 (1998).

31. D. Semrau, E. Sillekens, R. I. Killey, and P. Bayvel, "Isrs gn model implementation," https://githubcom/dsemrau/ISRSGNmodel (2019).

32. C. E. Shannon, "A mathematical theory of communication," Bell Syst. Tech. J. 27, 379-423 (1948).

33. C. R. Giles and E. Desurvire, "Modeling erbium-doped fiber amplifiers," J. Light. Technol. 9, 271-283 (1991).

34. A. Ferrari, A. Napoli, J. K. Fischer, N. Costa, J. Pedro, N. Sambo, E. Pincemin, B. Sommerkohrn-Krombholz, and V. Curri, "Upgrade capacity scenarios enabled by multi-band optical systems," in 201921st International Conference on Transparent Optical Networks (ICTON), (2019), pp. 1-4.

35. I. Roberts, J. M. Kahn, and D. Boertjes, "Convex channel power optimization in nonlinear wdm systems using gaussian noise model," J. Light. Technol. 34, 3212-3222 (2016).

36. S. Boyd and L. Vandenberghe, Convex Optimization (Cambridge, 2004).

37. R. Fletcher, Practical Methods of Optimization (John Wiley and Sons, 1987). 